CREAT. MATH. INFORM.

Volume 29 (2020), No. 2,

Pages 231 - 236

\title{
Generalized semi-open sets via ideals in topological space
}

\author{
RITU SEN
}

ABSTRACT. In this paper we have introduced a new type of sets termed as $\hat{\mu}$-open sets which unifies semiopen sets and discussed some of its properties. We have also introduced another type of weak open sets termed as $\mathcal{I}_{\hat{\mu}}$-open sets depending on a GT as well as an ideal on a topological space. Finally the concept of weakly $\mathcal{I}_{\hat{\tau}}$-open sets are investigated.

\section{INTRODUCTION}

The concept of ideal on topological spaces was studied by Kuratowski [11] and Vaidyanat-haswamy [17] which is one of the important area of research in the branch of mathematics. After then different mathematicians applied the concept of ideals in topological spaces (see $[2,8,9,10,14,16,17])$. In the past few years mathematicians turned their attention towards the generalized open sets (see $[3,4,6,15,16]$ for details). Our aim in this paper is to use the concept of ideals in the generalized topology introduced by A. Császár. We recall some notions defined in [4].

Let $\exp X$ denotes the power set of a non-empty set $X$. A class $\mu(\subseteq \exp X)$ is called a generalized topology [4], (briefly, GT) if $\varnothing \in \mu$ and $\mu$ is closed under arbitrary union. The elements of $\mu$ are called $\mu$-open sets and the complement of $\mu$-open sets are known as $\mu$-closed sets. A set $X$ with a GT $\mu$ on it is known as a generalized topological space (briefly, GTS) and is denoted by $(X, \mu)$. A GT $\mu$ is said to be a quasi topology (briefly QT) [5] if $M, M^{\prime} \in \mu$ implies $M \cap M^{\prime} \in \mu$. The pair $(X, \mu)$ is said to be a QTS if $\mu$ is a QT on $X$.

For any $A \subseteq X$, the generalized $\mu$-closure of $A$ is denoted by $c_{\mu}(A)$ and is defined by $c_{\mu}(A)=\cap\{F: F$ is $\mu$-closed and $A \subseteq F\}$, similarly $i_{\mu}(A)=\cup\{U: U \subseteq A$ and $U \in \mu\}$ (see $[4,6])$. Throughout the paper $\mu, \lambda$ will always mean GT on the respective sets.

An ideal [11] $\mathcal{I}$ on a topological space $(X, \tau)$ is a non-empty collection of subsets of $X$ with the following properties : (i) $A \subseteq B$ and $B \in \mathcal{I} \Rightarrow A \in \mathcal{I}$ (ii) $A \in \mathcal{I}, B \in \mathcal{I} \Rightarrow$ $A \cup B \in \mathcal{I}$. A topological space $(X, \tau)$ with an ideal $\mathcal{I}$ is denoted by $(X, \tau, \mathcal{I})$ and known as an ideal topological space.

\section{Properties of $\hat{\mu}$-OPEN, $\mathcal{I}_{\hat{\mu}}$-OPEN, AND WEAKLy $\mathcal{I}_{\hat{\tau}}$-OPEN SETS}

Definition 2.1. Let $\mu$ be a GT on a topological space $(X, \tau)$. A subset $A$ of $X$ is called $\hat{\mu}$-open if $A \subseteq c_{\mu}(\operatorname{int}(A))$.

Theorem 2.1. Let $\mu$ be a GT on a topological space $(X, \tau)$. A subset $A$ of $X$ is $\hat{\mu}$-open if and only if there exists an open set $U$ such that $U \subseteq A \subseteq c_{\mu}(U)$.

Proof. Let $A$ be a $\hat{\mu}$-open set. Then $A \subseteq c_{\mu}(\operatorname{int}(A))$. Let $U=\operatorname{int}(A)$. Then $U$ is an open set and $U \subseteq A \subseteq c_{\mu}(\operatorname{int}(A))=c_{\mu}(U)$.

Conversely, let there be an open set $U$ such that $U \subseteq A \subseteq c_{\mu}(U)$. Now $U \subseteq A \Rightarrow U \subseteq$ $\operatorname{int}(A) \Rightarrow c_{\mu}(U) \subseteq c_{\mu}(\operatorname{int}(A))$. Thus $A \subseteq c_{\mu}(\operatorname{int}(A))$.

Key words and phrases. $\mu$-open set, ideal, $\hat{\mu}$-open set, $\mathcal{I}_{\hat{\mu}}$-open set, weakly $\mathcal{I}_{\hat{\tau}}$-open set. 
Remark 2.1. Let $\mu$ be a GT on a topological space $(X, \tau)$. If

(i) $\mu=\tau$, then a $\hat{\mu}$-open set reduces to a semi-open set.

(ii) every open set is $\hat{\mu}$-open.

(iii) If $\lambda$ be any other GT on $X$ with $\mu \subseteq \lambda$, then every $\hat{\lambda}$-open set is $\hat{\mu}$-open.

Remark 2.2. Let $\mu$ be a GT on a topological space $(X, \tau)$. Then the collection of all $\hat{\mu}$-open sets forms a GT on $X$.

Proof. Clearly $\varnothing$ is a $\hat{\mu}$-open set. Let $\left\{A_{\alpha}: \alpha \in \Lambda\right\}$ be a family of $\hat{\mu}$-open sets. Then there exist open sets $U_{\alpha}$ such that $U_{\alpha} \subseteq A_{\alpha} \subseteq c_{\mu}\left(U_{\alpha}\right)$ for each $\alpha \in \Lambda$. Thus $\cup\left\{U_{\alpha}: \alpha \in \Lambda\right\}=U$ (say) $\subseteq \cup\left\{A_{\alpha}: \alpha \in \Lambda\right\} \subseteq c_{\mu}(U)$, where $U$ is open showing that the union of $\hat{\mu}$-open sets is a $\hat{\mu}$-open set.

Example 2.1. (a) Let $X=\{a, b, c\}, \tau=\{\varnothing,\{a\},\{a, c\}, X\}$ and $\mu=\{\varnothing,\{a, b\},\{a, c\}, X\}$. Then $\mu$ is a GT on the topological space $(X, \tau)$. It can be checked easily that $\{a, b\}$ is a $\hat{\mu}$-open set which is not an open set.

(b) Let $X=\{a, b, c, d\}, \mu=\{\varnothing,\{a\},\{a, b\},\{a, b, c\}\}$ and $\tau=\{\varnothing,\{a\},\{c\},\{a, c\},\{a, b\}$, $\{c, d\},\{a, b, c\},\{a, c, d\}, X\}$. Then $\mu$ is a GT on the topological space $(X, \tau)$. It can be easily verified that $\{a, d\}$ and $\{c, d\}$ are both $\hat{\mu}$-open but their intersection $\{d\}$ is not so.

Theorem 2.2. Let $\mu$ be a GT on a topological space $(X, \tau)$ and $A$ be a $\hat{\mu}$-open set such that $A \subseteq B \subseteq c_{\mu}(A)$. Then $B$ is also a $\hat{\mu}$-open set.

Proof. As $A$ is $\hat{\mu}$-open, there exists an open set $U$ such that $U \subseteq A \subseteq c_{\mu}(U)$. Thus $U \subseteq B$. Also $c_{\mu}(A) \subseteq c_{\mu}(U) \Rightarrow B \subseteq c_{\mu}(U)$. Thus $U \subseteq B \subseteq c_{\mu}(U)$. Thus $B$ is $\hat{\mu}$-open.

Definition 2.2. Let $\mu$ be a GT on an ideal topological space $(X, \tau, \mathcal{I})$. A subset $A$ of $X$ is called $\mathcal{I}_{\hat{\mu}}$-open if there exists an open set $U$ such that $U \backslash A \in \mathcal{I}$ and $A \backslash c_{\mu}(U) \in \mathcal{I}$.

If $A \in \mathcal{I}$, then $A$ is an $\mathcal{I}_{\hat{\mu}}$-open set and also by Theorem 2.1, every $\hat{\mu}$-open set (hence every open set) is $\mathcal{I}_{\hat{\mu}}$-open for any ideal $\mathcal{I}$ on $X$. Also note that if we take $\mu=\tau$, then $\mathcal{I}_{\hat{\mu}}$-open set reduces to $\mathcal{I}$-semi-open set [13].

Example 2.2. (a) Let $X=\{a, b, c\}, \mu=\{\varnothing,\{a\},\{a, b\},\{a, c\},\{b, c\}, X\}, \tau=\{\varnothing,\{a\},\{a, b\}$, $\{a, c\}, X\}$ and $\mathcal{I}=\{\varnothing,\{a\},\{b\},\{a, b\}\}$. Then $\mu$ is a GT on the ideal topological space $(X, \tau, \mathcal{I})$. It can be verified that $\{b\}$ is $\mathcal{I}_{\hat{\mu}}$-open but not $\hat{\mu}$-open.

(b) Let $\mathbb{R}$ be the set of reals, $\mathbb{Q}$ be the set of rationals and $\mathbb{I}$ be the set of irrationals. Consider $\mathcal{I}=\{A \subseteq \mathbb{R}: A$ is finite $\}$ and $\mu=\{\varnothing, \mathbb{I}, \mathbb{R}\}$. Then $\mu$ is a GT on the ideal topological space $\left(\mathbb{R}, \tau_{u}, \mathcal{I}\right)$, where $\tau_{u}$ denotes the usual topology on $\mathbb{R}$. We note that for all $x \in \mathbb{Q},\{x\}$ is an $\mathcal{I}_{\hat{\mu}}$-open set as $\{x\} \in \mathcal{I}$ but $\mathbb{Q}=\cup\{\{x\}: x \in \mathbb{Q}\}$ is not $\mathcal{I}_{\hat{\mu}}$-open.

(c) Let us consider $X=\{a, b, c, d\}, \mu=\{\varnothing,\{a, b, c\},\{b, c, d\},\{a, c, d\}, X\}, \tau=\{\varnothing,\{a\}$, $\{a, b\},\{a, c\},\{a, b, c\}, X\}$ and $\mathcal{I}=\{\varnothing,\{c\}\}$. It can be checked that $\{a, b, c\}$ and $\{b, c, d\}$ are both $\mathcal{I}_{\hat{\mu}}$-open but their intersection $\{b, c\}$ is not so.

Theorem 2.3. Let $\mu$ be a GT on an ideal topological space $(X, \tau, \mathcal{I})$ where $\mathcal{I}$ is not countably additive. Then $\mathcal{I}$ is a minimal ideal on $X$ i.e., $\mathcal{I}=\{\varnothing\}$ if and only if the concept of $\hat{\mu}$-openness and $\mathcal{I}_{\hat{\mu}}$-openness are the same.

Proof. Suppose that $\mathcal{I}=\{\varnothing\}$. It is sufficient to show that whenever $A$ is an $\mathcal{I}_{\hat{\mu}}$-open set it is $\hat{\mu}$-open. Indeed, if $A$ is $\mathcal{I}_{\hat{\mu}}$-open, then there exists an open set $U$ such that $U \backslash A$, $A \backslash c_{\mu}(U) \in \mathcal{I}=\{\varnothing\}$ and so $U \subseteq A \subseteq c_{\mu}(U)$ proving $A$ to be a $\hat{\mu}$-open set (by Theorem 2.1).

Conversely, whenever a set is $\mathcal{I}_{\hat{\mu}}$-open then it is $\hat{\mu}$-open. Let $A \in \mathcal{I}$. Then $A$ is an $\mathcal{I}_{\hat{\mu}}$ open set and hence by the assumption $A$ is a $\hat{\mu}$-open set. Thus there is an open set $V_{1}$ such that $V_{1} \subseteq A \subseteq c_{\mu}\left(V_{1}\right)$. Then $V_{1} \in \mathcal{I}$ (as $V_{1} \subseteq A$ and $A \in \mathcal{I}$ ). Thus $A \cup V_{1} \in \mathcal{I}$. By the similar 
argument as earlier $A \cup V_{1}$ is also $\hat{\mu}$-open. Thus there exists an open set $V_{2}$ such that $V_{2} \subseteq$ $A \cup V_{1} \subseteq c_{\mu}\left(V_{2}\right)$. Similarly, there exists an open set $V_{3}$ such that $V_{3} \subseteq A \cup V_{1} \cup V_{2} \subseteq c_{\mu}\left(V_{3}\right)$. Continuing in this way we can obtain an infinite sequence of open sets $V_{1}, V_{2}, V_{3}, \ldots$ such that $A \cup V_{1} \cup V_{2} \cup V_{3} \cup \ldots \in \mathcal{I}$. But this is not possible as $\mathcal{I}$ is not countably additive. Thus, it must be the case that $V_{1}=\varnothing$ (similarly for the other $V_{i}^{\prime}$ s). Thus $c_{\mu}\left(V_{1}\right)=\varnothing$. Thus $A=\varnothing$ (as $\left.V_{1} \subseteq A \subseteq c_{\mu}\left(V_{1}\right)\right)$. This shows that $\mathcal{I}=\varnothing$.

Remark 2.3. Let $\mathcal{I}$ and $\mathcal{I}^{\prime}$ be two ideals on a topological space $(X, \tau)$ and $\mu$ be a GT on $X$. If $\mathcal{I} \subseteq \mathcal{I}^{\prime}$, then every $\mathcal{I}_{\hat{\mu}}$-open set is $\mathcal{I}_{\hat{\mu}}^{\prime}$-open (see Definition 2.2) and hence if $A$ is $\left(\mathcal{I} \cap \mathcal{I}^{\prime}\right)_{\hat{\mu}}$-open, then it is $\mathcal{I}_{\hat{\mu}}$-open as well as $\mathcal{I}_{\hat{\mu}}^{\prime}$-open.

Proposition 2.1. Let $\mu$ be a GT on an ideal topological space $(X, \tau, \mathcal{I})$. The union of finite number of $\mathcal{I}_{\hat{\mu}}$-open sets is an $\mathcal{I}_{\hat{\mu}}$-open set.

Proof. Let $A$ and $B$ be two $\mathcal{I}_{\hat{\mu}}$-open sets. Then there exist two open sets $G$ and $H$ such that $G \backslash A \in \mathcal{I}, A \backslash c_{\mu}(G) \in \mathcal{I}, H \backslash B \in \mathcal{I}, B \backslash c_{\mu}(H) \in \mathcal{I}$. Let $U=G \cup H$ and observe that $U \backslash(A \cup B) \subseteq((G \backslash A) \backslash B) \cup((H \backslash B) \backslash A) \in \mathcal{I}$. Also $A \cup B \backslash c_{\mu}(G \cup H) \subseteq$ $\left(\left(A \backslash c_{\mu}(G) \backslash c_{\mu}(H)\right) \cup\left(\left(B \backslash c_{\mu}(H)\right) \backslash c_{\mu}(G)\right) \in \mathcal{I}\right.$. Thus $A \cup B$ is $\mathcal{I}_{\hat{\mu}}$-open.

Proposition 2.2. Let $\mu$ be a GT on an ideal topological space $(X, \tau, \mathcal{I})$ and suppose that there exists a $\mu$-dense open subset $A \in \mathcal{I}$. Then every subset $B$ of $X$ is $\mathcal{I}_{\hat{\mu}}$-open.

Proof. Let $B$ be any subset of $X$. Note that (as $\mathcal{I}$ is an ideal, $A \in \mathcal{I}, A \backslash B \subseteq A$ ), we shall have $A \backslash B \in \mathcal{I}$. Put $U=A$. Then $U \backslash B=A \backslash B \in \mathcal{I}$ and $B \backslash c_{\mu}(U)=B \backslash c_{\mu}(A)=B \backslash X=$ $\varnothing \in \mathcal{I}$. Consequently, $B$ is $\mathcal{I}_{\hat{\mu}}$-open.

Example 2.3. Let $X=\{a, b, c\}, \tau=\{\varnothing,\{a\},\{a, b\}, X\}$ be the topology on $X, \mathcal{I}=\{\varnothing,\{c\}\}$ and $\mu=\{\varnothing,\{a, b\},\{a, c\}, X\}$. Then $\mu$ is a GT on the ideal topological space $(X, \tau, \mathcal{I})$. It is easy to check that $c_{\mu}(\{a\})=X$ where $\{a\} \notin \mathcal{I}$. It can be checked that $\{b\}$ is not an $\mathcal{I}_{\hat{\mu}}$-open set.

Proposition 2.3. Let $\mu$ be a GT on an ideal topological space $(X, \tau, \mathcal{I})$ and $A$ be an open set such that $A \subseteq B \subseteq c_{\mu}(A)$. Then $B$ is an $\mathcal{I}_{\hat{\mu}}$-open set.

Proof. Obvious.

Proposition 2.4. Let $\mu$ be a GT on an ideal topological space $(X, \tau, \mathcal{I})$ where every non-empty open subset is $\mu$-dense in $(X, \tau)$. Then for any subset $A$ of $X$,

(a) if $A$ is $\mathcal{I}_{\hat{\mu}}$-open with $A \notin \mathcal{I}$, then

(i) $A \subseteq B$ implies $B$ is $\mathcal{I}_{\hat{\mu}}$-open.

(ii) $A \cup B$ is $\mathcal{I}_{\hat{\mu}}$-open for any subset $B$ of $X$.

(b) Moreover, if the collection of open subsets of $X$ satisfies finite intersection property and $A, B \notin$ $\mathcal{I}$ be two $\mathcal{I}_{\hat{\mu}}$-open sets, then $A \cap B$ is also an $\mathcal{I}_{\hat{\mu}}$-open set.

Proof. (a)(i) Suppose that $A$ is $\mathcal{I}_{\hat{\mu}}$-open and $A \subseteq B$. Then there is an open set $G$ such that $G \backslash A \in \mathcal{I}$ and $A \backslash c_{\mu}(G) \in \mathcal{I}$. We first observe that $G \neq \varnothing$ for otherwise, $c_{\mu}(G)=\varnothing$ (and $A \in \mathcal{I}$ ). Since $A \subseteq B$, we have $G \backslash B \subseteq G \backslash A \in \mathcal{I}$ and $B \backslash c_{\mu}(G)=B \backslash X=\varnothing \in \mathcal{I}$. Thus $B$ is $\mathcal{I}_{\hat{\mu}}$-open.

(ii) As $A \subseteq A \cup B$, (ii) follows directly from (i).

(b) Let $A$ and $B$ be two $\mathcal{I}_{\hat{\mu}}$-open sets. If $A \cap B=\varnothing$, then the proof is trivial. We assume therefore that $A \cap B \neq \varnothing$. By assumption there exist two open sets $G$ and $H$ such that $G \backslash A \in \mathcal{I}, A \backslash c_{\mu}(G) \in \mathcal{I}, H \backslash B \in \mathcal{I}, B \backslash c_{\mu}(H) \in \mathcal{I}$. Consider the open set $G \cap H$ which is non-empty. Since $G \cap H \backslash(A \cap B)=((G \backslash A) \cap H) \cup((H \backslash B) \cap G) \in \mathcal{I}$, $(A \cap B) \backslash c_{\mu}(G \cap H)=(A \cap B) \backslash X=\varnothing \in \mathcal{I}$, thus $A \cap B$ is $\mathcal{I}_{\hat{\mu}}$-open. 
Example 2.4. Let $X=\{a, b, c\}, \mathcal{I}=\{\varnothing,\{b\}\}, \tau=\{\varnothing,\{a\},\{c\},\{a, c\}, X\}$ and $\mu=$ $\{\varnothing,\{a, c\}, X\}$. Then $\mu$ is a GT on the ideal topological space $(X, \tau)$ such that every nonempty open set is $\mu$-dense in $(X, \tau)$. It can be checked that $\{b\}$ is an $\mathcal{I}_{\hat{\mu}}$-open set but $\{b, c\}$ is not so.

Proposition 2.5. Let $\mu$ be a $Q T$ on an ideal topological space $(X, \tau, \mathcal{I})$ with $\tau \subseteq \mu$ and every non-empty open subset is $\mu$-dense in $(X, \tau)$. A subset $A$ which is not $\mu$-dense is $\overline{\mathcal{I}}_{\hat{\mu}}$-open if and only if $c_{\mu}(A)$ is $\mathcal{I}_{\hat{\mu}}$-open.

Proof. Let $A$ be $\mathcal{I}_{\hat{\mu}}$-open. Then as $A \subseteq c_{\mu}(A)$, by Proposition 2.4(i), $c_{\mu}(A)$ is also $\mathcal{I}_{\hat{\mu}}$-open (if $A \notin \mathcal{I}$ ). For $A \in \mathcal{I}$, we proceed as follows: As $A$ is $\mathcal{I}_{\hat{\mu}}$-open, there exists an open set $U$ such that $U \backslash A$ and $A \backslash c_{\mu}(U)$ are both in $\mathcal{I}$ which implies that $(U \backslash A) \cup A=U \cup A \in \mathcal{I}$. Thus $U \backslash c_{\mu}(A) \in \mathcal{I}$ (as $\left.U \backslash c_{\mu}(A) \subseteq U \subseteq U \cup A\right)$. Also, $c_{\mu}(A) \backslash c_{\mu}(U)=c_{\mu}(A) \backslash X=\varnothing \in \mathcal{I}$.

Conversely, suppose that $c_{\mu}(A)$ is $\mathcal{I}_{\hat{\mu}}$-open. Then there exists an open set $G$ such that $G \backslash c_{\mu}(A) \in \mathcal{I}$ and $c_{\mu}(A) \backslash c_{\mu}(G) \in \mathcal{I}$. If $G=\varnothing$, then $c_{\mu}(A) \backslash c_{\mu}(G)=c_{\mu}(A) \in \mathcal{I} \Rightarrow A \in \mathcal{I}$. Thus $A$ is $\mathcal{I}_{\hat{\mu}}$-open. If $G$ is non-empty, consider the $\mu$-open set $H=G \backslash c_{\mu}(A)=G \cap(X \backslash$ $\left.c_{\mu}(A)\right) \in \mathcal{I}$. Again, $H \backslash A=G \cap\left(X \backslash c_{\mu}(A)\right) \cap(X \backslash A) \subseteq G \cap\left(X \backslash c_{\mu}(A)\right) \in \mathcal{I}$. Thus $H \backslash A \in \mathcal{I}$ and $A \backslash c_{\mu}(H)=A \backslash c_{\mu}\left(G \cap\left(X \backslash c_{\mu}(A)\right)\right)=A \backslash X=\varnothing$. This shows that $A$ is $\mathcal{I}_{\hat{\mu}}$-open.

Theorem 2.4. Let $\mu$ be a GT on an ideal topological space $(X, \tau, \mathcal{I})$. Then $X \backslash A$ is $\mathcal{I}_{\hat{\mu}}$-open if and only if there exists a closed set $F$ such that $i_{\mu}(F) \backslash A \in \mathcal{I}$ and $A \backslash F \in \mathcal{I}$.

Proof. First suppose that $X \backslash A$ is $\mathcal{I}_{\hat{\mu}}$-open. Then there exists an open set $G$ such that $G \backslash(X \backslash A)=A \backslash(X \backslash G) \in \mathcal{I}$ and $(X \backslash A) \backslash c_{\mu}(G)=i_{\mu}(X \backslash G) \backslash A \in \mathcal{I}$. Let $F=X \backslash G$. Then $F$ is closed and the rest follows. The converse part can be done similarly by taking $G=X \backslash F$.

Definition 2.3. Let $(X, \tau, \mathcal{I})$ be an ideal topological space. A subset $A$ of $X$ is called weakly $\mathcal{I}_{\hat{\tau}}$-open if $A=\varnothing$ or if $A \neq \varnothing$, there exists a non-empty open set $U$ such that $U \backslash A \in \mathcal{I}$. The complement of a weakly $\mathcal{I}_{\hat{\tau}}$-open set is termed as weakly $\mathcal{I}_{\hat{\tau}}$-closed set.

It follows that for an ideal topological space $(X, \tau, \mathcal{I})$ with a GT $\mu$ on $X$, any $\mathcal{I}_{\hat{\mu}}$-open set (hence open set) is weakly $\mathcal{I}_{\hat{\tau}}$-open but the converse is false follows from the next example.

Example 2.5. (a) Consider $X=\{a, b, c, d\}, \tau=\mu=\{\varnothing,\{a, b\},\{c, d\}, X\}$ and $\mathcal{I}=\{\varnothing,\{a\}\}$. Then $(X, \tau, \mathcal{I})$ is an ideal topological space. It is easy to see that $\{b, c\}$ is a weakly $\mathcal{I}_{\hat{\tau}}$-open set but not $\mathcal{I}_{\hat{\mu}}$-open.

(b) Consider $X=\{a, b, c, d\}, \tau=\{\varnothing,\{a, b\},\{c, d\}, X\}$ and $\mathcal{I}=\{\varnothing,\{d\}\}$. Then $\mathcal{I}$ is an ideal on the topological space $(X, \tau)$. It is easy to see that $\{a, b\}$ and $\{a, c\}$ are two weakly $\mathcal{I}_{\hat{\tau}}$-open sets but their intersection $\{a\}$ is not so.

Proposition 2.6. Let $(X, \tau, \mathcal{I})$ be an ideal topological space. Then the collection of all weakly $\mathcal{I}_{\hat{\tau}}$-open sets form a GT on $X$.

Proof. $\varnothing$ is clearly a weakly $\mathcal{I}_{\hat{\tau}}$-open set. Let $\left\{A_{\alpha}: \alpha \in \Lambda\right\}$ be a collection of weakly $\mathcal{I}_{\hat{\tau}}$ open sets. Then for each $\alpha \in \Lambda$, there exists a non-empty open set $U_{\alpha}$ such that $U_{\alpha} \backslash A_{\alpha} \in \mathcal{I}$. But $U_{\alpha} \backslash \cup\left\{A_{\alpha}: \alpha \in \Lambda\right\} \subseteq U_{\alpha} \backslash A_{\alpha}$. Thus $U_{\alpha} \backslash \cup\left\{A_{\alpha}: \alpha \in \Lambda\right\} \in \mathcal{I}$. Hence $\cup\left\{A_{\alpha}: \alpha \in \Lambda\right\}$ is a weakly $\mathcal{I}_{\hat{\tau}}$-open set.

Theorem 2.5. Let $(X, \tau, \mathcal{I})$ an ideal topological space. Then a non-empty subset $A$ of $X$ is weakly $\mathcal{I}_{\hat{\tau}}$-open if and only if there exist a non-empty open set $U$ and a set $C$ in $\mathcal{I}$ such that $U \backslash C \subseteq A$. 
Proof. Let $A$ be a non-empty weakly $\mathcal{I}_{\hat{\tau}}$-open subset of $X$. Then there exists a non-empty open set such that $U \backslash A \in \mathcal{I}$. Let $C=U \backslash A=U \cap(X \backslash A)$. Then $U \backslash C \subseteq A$.

Conversely, let there exist an open set $U$ and $C$ in $\mathcal{I}$ such that $U \backslash C \leqq \bar{A}$. Then $U \backslash A \subseteq$ $U \cap C \in \mathcal{I}$ (as $C \in \mathcal{I}$ ). Thus $U \backslash A \in \mathcal{I}$.

Theorem 2.6. Let $(X, \tau, \mathcal{I})$ be an ideal topological space. Then if a subset $A$ of $X$ is weakly $\mathcal{I}_{\hat{\tau}}$-closed, then $A \subseteq K \cup B$ for some closed set $K$ of $X$ and $B \in \mathcal{I}$.

Proof. Let $A$ be a weakly $\mathcal{I}_{\hat{\tau}}$-closed set. Then $X \backslash A$ is a weakly $\mathcal{I}_{\hat{\tau}}$-open set. If $X \backslash A=\varnothing$, then $A=X$. Thus $A=X \cup \varnothing$. If $A \neq X$, then there is a non-empty open set $U$ and $B \in \mathcal{I}$ such that $U \backslash B \subseteq X \backslash A$. So $A \subseteq X \backslash(U \backslash B)=(X \backslash U) \cup B=K \cup B$ where $K=X \backslash U$ which is a closed set and $B \in \mathcal{I}$.

Example 2.6. Let $X=\{a, b, c, d\}, \mathcal{I}=\{\varnothing,\{d\}\}$ and $\tau=\{\varnothing,\{a\},\{a, b\},\{a, c\},\{a, b, c\}, X\}$. Then $\{a, d\} \subseteq X \cup\{d\}$, where $X$ is $\mu$-closed and $\{d\} \in \mathcal{I}$ but $\{a, d\}$ is not a weakly $\mathcal{I}_{\hat{\tau}}$ closed set.

Remark 2.4. Let $(X, \tau, \mathcal{I})$ be an ideal topological space. If $\varnothing \neq A \varsubsetneqq B$ and $A$ is a weakly $\mathcal{I}_{\hat{\tau}}$-open set, then so is $B$ (by Definition 2.3). Thus if $A$ is a weakly $\mathcal{I}_{\hat{\tau}}$-open set so is $A \cup B$, for any subset $B$ of $X$. In particular, $\operatorname{cl}(A)$ is a weakly $\mathcal{I}_{\hat{\tau}}$-open set if $A$ is so but the converse is not true as is seen from the following example.

Example 2.7. Let $X=\{a, b, c, d\}$ and $\tau=\{\varnothing,\{a, c\},\{b, d\}, X\}$ and $\mathcal{I}=\{\varnothing,\{b\}\}$. It is easy to verify that $\{a\}$ is not a weakly $\mathcal{I}_{\hat{\tau}}$-open set though $\operatorname{cl}(\{a\})$ is weakly $\mathcal{I}_{\hat{\tau}}$-open.

Theorem 2.7. Let $(X, \tau, \mathcal{I})$ be an ideal topological space such that $\{a\} \in \tau \cap \mathcal{I}$ for some $a \in X$. Then every subset of $X$ is weakly $\mathcal{I}_{\hat{\tau}}$-open.

Proof. Suppose $\{b\} \subseteq X$. Then either $\{a\} \backslash\{b\}=\varnothing \in \mathcal{I}$ (if $b=a$ ) or $\{a\} \backslash\{b\}=\{a\} \in \mathcal{I}$ (if $a \neq b)$, where $\{a\} \in \tau$. Thus $\{b\}$ is weakly $\mathcal{I}_{\hat{\tau}}$-open. Thus by Proposition 2.6, any subset of $X$ is weakly $\mathcal{I}_{\hat{\tau}}$-open.

Theorem 2.8. Let $(X, \tau, \mathcal{I})$ be an ideal topological space $(X, \tau, \mathcal{I})$. Then the collection of weakly $\mathcal{I}_{\hat{\tau}}$-open sets form a topology on $X$.

Proof. Due to Proposition 2.6, we have only to show that $X$ is weakly $\mathcal{I}_{\hat{\tau}}$-open and that the intersection of two weakly $\mathcal{I}_{\hat{\tau}}$-open sets is so. Since $X \in \tau, X$ is weakly $\mathcal{I}_{\hat{\tau}}$-open. Let $A$ and $B$ be two weakly $\mathcal{I}_{\hat{\tau}}$-open sets. Then there exist non-empty open sets $U$ and $V$ such that $U \backslash A \in \mathcal{I}$ and $V \backslash B \in \mathcal{I}$. Then $(U \cap V) \backslash(A \cap B)=[(U \backslash A) \cap V] \cup[U \cap(V \backslash B)] \in \mathcal{I}$. Thus $A \cap B$ is a weakly $\mathcal{I}_{\hat{\tau}}$-open set.

Theorem 2.9. Let $(X, \tau, \mathcal{I})$ an ideal topological space such that the open sets of $X$ satisfies finite intersection property, where $\mathcal{I} \neq\{\varnothing\}$. Let $A$ be a subset of $X$ such that $\operatorname{cl}(A) \neq X$. Then $A$ is weakly $\mathcal{I}_{\hat{\tau}}$-open if and only if $\operatorname{cl}(A)$ is so.

Proof. We first observe that if $A(\neq \varnothing)$ is weakly $\mathcal{I}_{\hat{\tau}}$-open and $A \subseteq B$, then $B$ is weakly $\mathcal{I}_{\hat{\tau}}$-open and thus $\operatorname{cl}(A)$ is weakly $\mathcal{I}_{\hat{\tau}}$-open (as $A \subseteq \operatorname{cl}(A)$ ).

Conversely, suppose that $\operatorname{cl}(A)$ is a weakly $\mathcal{I}_{\hat{\tau}}$-open set. If $\operatorname{cl}(A)=\varnothing$, then $A=\varnothing$. Thus $A$ is a weakly $\mathcal{I}_{\hat{\tau}}$-open set. If $\operatorname{cl}(A) \neq \varnothing$, then $U \backslash \operatorname{cl}(A) \in \mathcal{I}$ for some non-empty open set $U$. Let $V=U \backslash \operatorname{cl}(A)$. Then $V \backslash A=U \backslash \operatorname{cl}(A) \in \mathcal{I}$. It is now sufficient to show that $V \neq \varnothing$. We note that $X \backslash \operatorname{cl}(A)$ and $U$ are non-empty open sets. Thus $(X \backslash \operatorname{cl}(A)) \cap U=V \neq \varnothing$. Thus $A$ is weakly $\mathcal{I}_{\hat{\tau}}$-open.

Example 2.8. Let $X=\{a, b\}, \tau=\{\varnothing, X\}$ and $\mathcal{I}=\{\varnothing,\{a\}\}$. Put $A=\{a\}$. Then $A$ is not a weakly $\mathcal{I}_{\hat{\tau}}$-open subset of $X$ such that $\operatorname{cl}(A)=X$. However, $\operatorname{cl}(A)$ is weakly $\mathcal{I}_{\hat{\tau}}$-open. 
Acknowledgement. The author is thankful to the referee for some comments for the improvement of the paper.

\section{REFERENCES}

[1] Abd El-Monsef, M. E., El-Deeb S. N. and Mahmoud, R. A., $\beta$-open sets and $\beta$-continuous mappings, Bulletin of the Faculty Science Assiut Univ., 12 (1983), 77-90

[2] Arenas, F. G., Dontchev, J. and Puertas, M. L., Idealization of some weak separation axioms, Acta Math. Hungar., 89 (2000), 47-53

[3] Császár, Á., Generalized open sets, Acta Math. Hungar., 75 (1997), 65-87

[4] Császár, Á., Generalized topology, generalized continuity, Acta Math. Hungar., 96 (2002), 351-357

[5] Császár, Á., Remarks on quasi topologies, Acta Math. Hungar., 119 (2008), 197-200

[6] Császár, Á., $\delta$ - and $\theta$-modifications of generalized topologies, Acta Math. Hungar., 120 (2008), 275-279

[7] Ekici, E., Generalized hyperconnectedness, Acta Math. Hungar., 133 (2011), No. 1-2, 140-147

[8] Ekici, E. and Noiri, T., *-extremally disconnected ideal topological spaces, Acta Math. Hungar., 122 (2009), 81-90

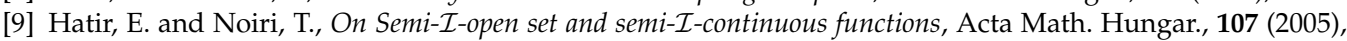
345-353

[10] Jankovic, D. and Hamlett, T. R., New topologies from old via Ideals, Amer. Math. Monthly, 97 (1990), 295-310

[11] Kuratowski, K., Topology, Vol-1, New York, Academic Press, 1966

[12] Levine, N., Semi-open sets and semi-continuity in topological spaces, Amer. Math. Monthly, 70 (1963), 36-41

[13] Michael, F., On semi-open sets with respect to an ideal, European Jour. Pure and Appl. Math., 6 (2013), No. 1, 53-58

[14] Mukherjee, M. N., Roy B. and Sen, R., On extension of topological spaces in terms of ideals, Topology and its Applications, 154 (2007), 3167-3172

[15] Roy, B., On weakly ( $\mu, \lambda$ )-open functions, Ukrainian Math. Jour., 66 (2015), No. 10, 1595-1602

[16] Shen, R. X., A note on generalized connectedness, Acta Math. Hungar., 122 (2009), 231-235

[17] Vaidyanathaswamy, R., The localization theory in set-topology, Proceedings of the Indian Acad. Sci., 20 (1945), $15-51$

DEPARTMENT OF MATHEMATICS

PRESIDENCY UNIVERSITY

86/1 College Street, Kolkata -700 073, W. B., India

Email address: ritu_sen29@yahoo.co.in 